\title{
Analysis of Internal Design Parameters to Minimize Geometrical Losses in Free-Space Optical Communication Link
}

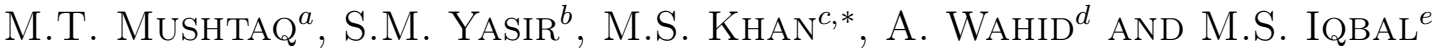 \\ ${ }^{a}$ School of Systems and Technology (SST), UMT Lahore, Pakistan \\ ${ }^{b}$ Department of Electrical Technology, Government Swedish Pakistani Institute of Technology, Gujrat, Pakistan \\ ${ }^{c}$ Department of Electrical Engineering, Faculty of Engineering, Lahore Leads University, Lahore, Pakistan \\ ${ }^{d}$ Department of Electronics, Government Swedish Pakistani Institute of Technology, Gujrat, Pakistan \\ ${ }^{e}$ Department of Electrical and Electronic Engineering, KOÇ University, Istanbul, Turkey
}

\begin{abstract}
During the last two decades, free-space optical links got considerable importance due to their benefits of higher data rates, license free-spectrum, easy and rapid deployment and mobility. Free-space optical links use carrier frequency in the range of $20 \mathrm{THz}$ to $375 \mathrm{THz}$ (in near infrared (IR) region and visible band in wavelengths) to establish a communication link for terrestrial communication, inter-satellite links, deep space links, ground-tosatellite and satellite-to-ground links. Free-space optical links are also useful for different military applications, disaster recovery and last mile access. However, despite of having all these advantages the performance of freespace optical links depends upon the atmospheric conditions and parameters of system design. Geometrical losses of free-space optical links are directly related to parameters of system design or internal parameters. In this paper we analyzed different parameters of system design to minimize the geometrical losses. We presented the analysis of internal design parameters like divergence angle, diameter of receiver aperture, diameter of transmitter aperture, link distance and suggested the suitable parameters of system design.
\end{abstract}

DOI: 10.12693/APhysPolA.134.275

PACS/topics: free-space optical, geometric loss, divergence angle, attenuation, link margin, link distance, received power, receiver aperture and transmitter aperture

\section{Introduction}

In the recent years tremendous developments have been observed in telecommunication technologies. Modern communication applications like high speed internet, video-conferencing, live streaming etc. have increased the demands of higher data rates and requires mobility and flexibility in the telecommunication networks. The demands in higher data rates of different telecommunication and multimedia services have led to congestion in spectrum of conventional radio frequency (RF) communication links and have raised the needs to shift the spectrum from RF to optical carrier. Optical carrier are free from spectrum licensing and therefore, became a natural and attractive alternative to RF links for capacity hungry communication applications. FSO links offers the advantages of license free communication, easy installation, immunity to electromagnetic interference and higher data rates $[1,2]$.

FSO links use optical signals to carry information from one point to another point passing through the atmosphere. The transmitted light signal experiences environmental effects like rain snow, smoke, fog, ambient temperature (scintillation effects) and aerosol particles suspended in the air [3-5].

*corresponding author; e-mail: msaeedbaloch@gmail.com3522
The performance of FSO links depend upon various factors like local weather conditions or environmental factors (channel conditions) and system design parameters. Local weather conditions or environmental factors are called as external parameters whereas the parameters of system design are called as internal parameters. Internal parameters are transmitted optical power, received optical power, selection of wavelength, divergence angle, diameter of transmitter and receiver aperture, link distance and receiver field of view (FoV).

In this paper, internal design parameters (divergence angle $\theta$, diameter of transmitter $d_{t}$, receiver aperture $d_{r}$ and link distance $L$ ) are analyzed to minimize the geometrical losses (GL).

Optical transmitter transmits the collimated beam of light. The width of transmitted beam increases with the link distance. The higher beam width causes to reduce the link margin at the receiver side which results in signal loss, reduction in signal-to-noise ratio (SNR) and increase in bit error rate (BER). On receiver side diameter of receiver aperture should be larger in size to receive all the information transmitted through optical carrier but on the same time larger receiver aperture results in noise from the ambient light. The geometrical losses for FSO links are given below in Eq. (1) [6]:

$$
\text { geometrical loss }=10 \log \left[\frac{d_{r}^{2}}{\left(d_{t}+(L \theta)\right)^{2}}\right] \text {. }
$$


Here $d_{t}$ represents diameter of the transmitter (in $\mathrm{mm}$ ), $d_{r}$ is the diameter of receiver (in $\mathrm{mm}$ ), $\theta$ is divergence angle of beam in mrad and $L$ is the length of the communication link in $m$. Geometrical losses are considered as constant losses because all internal design parameters remain constant. The total attenuation is a combination of atmospheric attenuation and geometric loss. Total attenuation is given in Eq. (2) [7]:

$$
\text { Attenuation }[\mathrm{dB} / \mathrm{km}]=\frac{d_{r}^{2}}{\left(d_{t}+(L \theta)\right)^{2}} \tau \text {, }
$$

whereas is total atmospheric attenuation. The total attenuation of link path $L$ can be calculated using Beer's law equation as given below in Eq. (3):

$$
\tau=\exp (\beta L) \mathrm{dB} / \mathrm{km},
$$

where $\beta$ is attenuation coefficient for both absorption and scattering and is expressed by Eq. (4):

$$
\beta=\beta_{\text {absorption }}+\beta_{\text {scattering. }} \text {. }
$$

\section{Simulations for geometric loss}

The value of geometric losses are calculated to determine its effects on the performance of FSO link. We selected four different designs for comparison at the link range of $5000 \mathrm{~m}$. The particular design specifications are shown in Table I. The implementation of the link was done with the help of components and devices available in the local market.

TABLE I

Illustration of design models with variation limits.

\begin{tabular}{l|c|c|c|c}
\hline \hline \multicolumn{1}{c|}{ Parameters } & Design model 1 & Design model 2 & Design model 3 & Design model 4 \\
\hline diameter of transmitter aperture $d_{t}$ & $0.08 \mathrm{~m}$ & $0.05 \mathrm{~m}$ & $0.010 \mathrm{~m}$ & $0.012 \mathrm{~m}$ \\
diameter of receiver aperture $d_{r}$ & $0.10 \mathrm{~m}$ & $0.08 \mathrm{~m}$ & $0.012 \mathrm{~m}$ & $0.015 \mathrm{~m}$ \\
divergence angle $\theta$ & $0.025 \mathrm{mrad}$ & $0.025 \mathrm{mrad}$ & $0.025 \mathrm{mrad}$ & $0.025 \mathrm{mrad}$ \\
link distance $L$ & $5000 \mathrm{~m}$ & $5000 \mathrm{~m}$ & $5000 \mathrm{~m}$ & $5000 \mathrm{~m}$
\end{tabular}

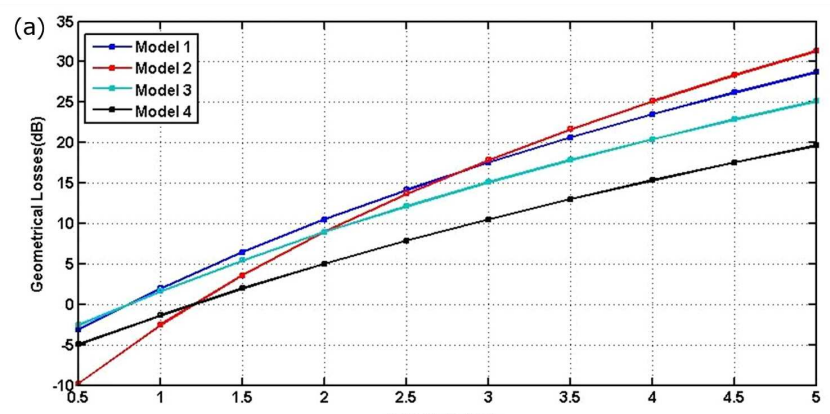

(b)

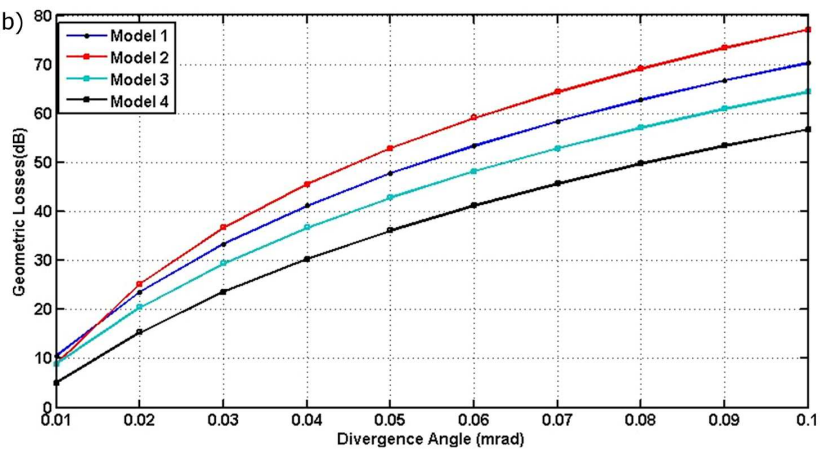

Fig. 1. Effect of link path and divergence angle on geometrical loss.

Figure 1a shows the relationship between geometric losses and link distance. Figure 1a clearly distinguishes that geometric loss $(\mathrm{dB})$ increases as the link path increases. Model 4 is observed to be a better option for design considerations due to minimum geometrical losses as compared to all other design model for a link distance of $5 \mathrm{~km}$ but it is not a good option where a link distance increases to $5 \mathrm{~km}$ or above because as the optical beam travelled through long turbulence channel, the beam footprint at the receiver spread requires larger diameter of receiver aperture.
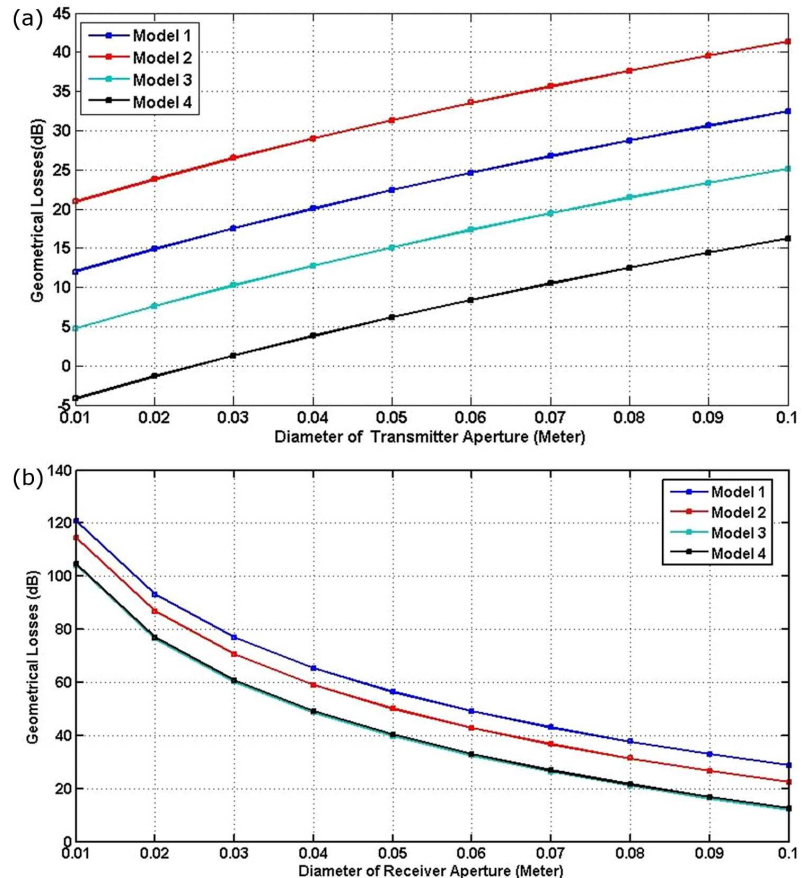

Fig. 2. Effect of diameter of transmitter aperture and receiver aperture on geometrical losses.

Figure $1 \mathrm{~b}$ shows the effect divergence angle on GL. Greater the divergence angle greater will be the beam front at the receiver side results in higher GL. Figure $2 \mathrm{a}$ illustrates the relationship between GL against diameter of transmitter aperture. GL has proportionate relationship with diameter of transmitter's aperture. The GL increases with increase in diameter of receiver aperture and increases for all design models (while keeping the other parameters constant e.g. link path $L$, divergence angle $\theta$, diameter of receiver aperture). Figure $2 \mathrm{~b}$ illustrates the relation between GL versus diameter 
of receiver's aperture $d_{r}$. Model design 4 is the most suitable model for selected design parameters.

\section{Conclusions}

Geometrical losses contribute towards signal losses and vary along with the link distance. These losses could be minimized by selecting small size of the diameter of the transmitter's aperture, appropriate size of the lens, and very small beam divergence angle at the receiver side. The diameter of the receiver aperture should be large in size as compared to the diameter of transmitter aperture but should not be large enough that ambient light may lump in the original signal and produce noise signals. The results are concluded here with the suggestion that for a FSO communication link of $5 \mathrm{~km}$ the diameter of transmitter aperture should be less than $5 \mathrm{~cm}$ with suitable large size diameter of receiver aperture $(15 \mathrm{~cm})$ and small divergence angle (less than $0.025 \mathrm{mrad}$ ). On the optical receiver side $\mathrm{FoV}$ is another important parameter which should be according to the size of beam while the greater FoV also produces the noise in the original signal.
Over all model design 4 observed to be the most suitable model under defined design parameters.

\section{References}

[1] X. Wang, M. Yao, X. Yi, Z. Liu, Z. Qiu, J. Mod. Opt. 62, 39 (2015).

[2] M.S. Awan, R. Nebuloni, C. Capsoni, L. CsurgaiHorváth, S.S. Muhammad, F. Nadeem, M.S. Khan, E. Leitgeb, Int. J. Satell. Commun. Network 29, 97 (2011).

[3] M. Al-Naboulsi, H. Sizun, F. de-Fornel, Opt. Eng. 43, 319 (2004).

[4] S. Mori, F.S. Marzano, J. Appl. Opt. 54, 6787 (2015).

[5] F. Nadeem, E. Leitgeb, J. Rad. Eng. 19, 223 (2010).

[6] I.I. Kim, E.J. Korevaar, SPIE Conf. Proc. 4530, 84 (2001).

[7] X. Tang,Ph.D. Thesis, University of Northumbria, Newcastle 2012. 